\title{
Boundary Effects on Diffusiophoresis of Cylindrical Particles in Nonelectrolyte Gradients
}

\author{
Huan J. Keh ${ }^{1}$ and Jung H. Hsu \\ Department of Chemical Engineering, National Taiwan University, Taipei 106-17, Taiwan, Republic of China
}

Received April 19, 1999; accepted October 14, 1999

The diffusiophoretic motion of a long circular cylinder in a transversely imposed gradient of a nonionic solute near a large plane wall parallel to its axis is analyzed. The range of the interaction between the solute and the solid surfaces is assumed to be small relative to the particle radius and to the gap width between the particle and the wall, but the polarization effect of the mobile solute in the thin diffuse layers adjacent to the solid surfaces caused by thestrong adsorption of the solute is incorporated. A normal flux of the solute and a slip velocity of the fluid at the outer edge of the diffuse layers are used as the boundary conditions for the fluid domain outside thediffuse layers. Through the use of cylindrical bipolar coordinates along with these boundary conditions, a set of transport equations governing this problem is solved in the quasisteady situation and the wall effects on the diffusiophoresis of the cylinder are computed for various cases. For the diffusiophoretic motion of a cylinder normal to a plane, the particle mobility decreases monotonically with the decrease of the distance of the particle axis from the wall. The stronger the polarization effect in the diffuse layer, the weaker the wall effect on the diffusiophoresis. The effect of the normal plane on the diffusiophoresis of a cylinder is much more significant than that for a sphere at the same separation. For the diffusiophoresis of a cylinder parallel to a plane, the boundary effect is a complicated function of the relevant parameters (not necessarily varies monotonically with the extent of separation) mainly due to the existence of a diffusio-osmotic flow caused by the tangential fluid velocity at the plane wall. $\odot 2000$ Academic Press

Key Words: diffusiophoresis in nonelectrolyte gradient; circular cylindrical particle; boundary effect; thin but polarized diffuse layer; diffusio-osmotic flow.

\section{INTRODUCTION}

A colloidal particle can be driven to move by a solute concentration gradient that interacts with the surface of the particle. This phenomenon, known as diffusiophoresis (1), has been demonstrated experimentally for both ionic (2) and nonionic (3) solutes. In a solution of nonelectrolyte solute with constant concentration gradient $\nabla C_{\infty}$, the diffusiophoretic velocity of a

\footnotetext{
${ }^{1}$ To whom correspondence should be addressed.
}

particle is (4)

$$
\mathbf{U}_{0}=\frac{k T}{\eta} L^{*} K \nabla C_{\infty}
$$

and there is no rotational motion of the particle. In the above equation, $L^{*}$ is a characteristic length for the particle-solute interaction (of order $1-10 \mathrm{~nm}$ ), $K$ is the Gibbs adsorption length characterizing the strength of the adsorption of the molecular solute ( $K$ and $L^{*}$ are defined by Eqs. [2.6a] and [2.6b]). $\eta$ is the fluid viscosity, $k$ is the Boltzmann constant, and $T$ is the absolute temperature. Equation [1.1] can be applied to an isolated rigid particle of arbitrary shape and size. However, its validity is based on the assumption that the local radii of curvature of the particle are much larger than the thickness of the particle-solute interaction layer (diffuse layer) at the particle surface and the effect of the "polarization" of the diffuse solute (the solute continually adsorbs at the upstream edge and desorbs at the downstream edge) in the interfacial layer surrounding the particle is negligible.

In the past decade, important advances have been made in the evaluation of the diffusiophoretic velocity of colloidal particles, relaxing this assumption. Anderson and Prieve (5) examined the diffusiophoresis of a colloidal sphere of radius $a$ with a thin but polarized diffuse layer at the particle surface when the solution is only slightly nonuniform in solute concentration on the length scale of $a\left(a\left|\nabla C_{\infty}\right| \ll C_{\infty}\right)$. Their result for the diffusiophoretic velocity of the particle is

$$
\mathbf{U}^{(0)}=\frac{k T}{\eta} L^{*} K\left(1+\frac{\beta}{a}\right)^{-1} \nabla C_{\infty},
$$

where the definition of $\beta$ is given by Eq. [2.5]. For a strongly adsorbing solute (e.g., a surfactant), the relaxation parameter $\beta / a$ (or $K / a$ ) can be much greater than unity. If all the adsorbed solute were "struck" to the surface of the particle (the diffuseness of the adsorption layer disappears), then $L^{*}=0$ and there would be no diffusiophoretic migration of the particle. In the limit of $\beta / a \rightarrow 0$ (very weak adsorption), the polarization of the diffuse solute in the interfacial layer vanishes and Eq. [1.2] reduces to Eq. [1.1]. It can be seen from Eqs. [1.1] and [1.2] that the effect of polarization of the diffuse layer is to lower the diffusiophoretic mobility of the particle. The reason for this consequence is that 
the transport of the solute inside the particle-solute interaction layer reduces the concentration gradient along the particle surface. The extension of Eq. [1.2] to the diffusiophoretic velocity of spheroidal (6) and cylindrical $(7,8)$ particles has also been derived. A remarkable feature was found that the diffusiophoretic velocity of a long circular cylinder in a transversely applied solute gradient is exactly the same as that of a sphere with an equal radius, given by Eq. [1.2].

In many real diffusiophoresis situations, colloidal particles are not isolated and the surrounding fluid is externally bounded by solid walls. Thus, it is important to determine if the presence of neighboring boundaries significantly affects the movement of particles. In the limiting case that Eq. [1.1] is applicable, the normalized velocity field of the unbounded fluid that is dragged by a particle during diffusiophoresis is the same as for electrophoresis of the dielectric particle with an infinitesimally thin electric double layer (9); thus, the boundary effects on electrophoresis, which have been investigated extensively in the past (10-12), can be utilized to interpret those on diffusiophoresis. An important result of these investigations is that the boundary effects on electrophoresis (and on diffusiophoresis) are much weaker than on sedimentation, because the disturbance to the fluid velocity field caused by a phoretic particle decays faster than that caused by a stokeslet.

When the polarization effect of the mobile solute in the diffuse layer adjacent to the solid surfaces is considered, the boundary effects on diffusiophoresis can be quite different from those on electrophoresis, due to the fact that the particle size and some other unique factors are involved in each transport mechanism (7). Through the use of a boundary collocation method, the diffusiophoresis and electrophoresis of a colloidal sphere with a thin but polarized diffuse layer in the direction normal to a plane wall have recently been examined (13). Numerical results of the wall-corrected migration velocities of the particle were presented for various cases. It was found that the particle velocity decreases steadily as the particle approaches the wall for both cases of diffusiophoresis in a nonelectrolyte gradient and of electrophoresis. Although the retardation effect produced by the wall on diffusiophoresis is a monotonic decreasing function of the relaxation parameter $\beta / a$, this effect on electrophoresis is a complicated function of the properties of the dielectric particle and surrounding electrolyte.

The objective of this paper is to determine the diffusiophoretic mobility of a long circular cylinder near a large plane wall parallel to its axis in transversely imposed solute gradients and to compare the results with those available for the corresponding diffusiophoresis of a sphere. The polarized diffuse layers are assumed to be thin compared with the radius of the particle and with the surface-to-surface spacing between the particle and the wall. A cylindrical bipolar coordinate system is used to solve the quasisteady problem. In the next section, we present the fundamental transport equations and boundary conditions which govern the solute concentration distribution and fluid flow field outside the thin polarized diffuse layers. Based on these linear transport equations and boundary conditions, the diffusiophoresis of a cylindrical particle in the direction perpendicular to its axis and to a plane wall is examined in Section 3. The closedform analytical solution for the wall-corrected diffusiophoretic mobility of the particle in a constant solute gradient is obtained in Eq. [3.16]. Section 4 provides the analysis of a complementary problem to that treated in Section 3, the diffusiophoretic motion of a cylinder in the direction normal to its axis and parallel to a plane wall. The general expressions for the translational and rotational velocities of the diffusiophoretic particle in this case (which allows the polarization of the diffuse layer adjacent to the parallel plane) are presented in Eq. [4.11].

\section{BASIC EQUATIONS FOR DIFFUSIOPHORETIC MOTION}

Consider the diffusiophoresis of a rigid particle of arbitrary shape in the vicinity of a solid boundary in a fluid solution containing an uncharged solute. This problem can be dealt with as a quasisteady state if both the Peclet and the Reynolds numbers are vanishingly small. It is assumed that the layer of interaction between the solute molecules and each fluid/solid interface is thin in comparison with the radii of curvature of the interface and the spacing between the particle and the boundary. Hence, the fluid phase can be divided into two regions: an "inner" region defined as the thin interaction layers adjacent to the interfaces and an "outer" region defined as the remainder of the fluid. In the outer region, the equations of conservation of the molecular solute and the fluid momentum can be expressed by the Laplace equation,

$$
\nabla^{2} C=0
$$

and the Stokes equations,

$$
\begin{aligned}
\eta \nabla^{2} \mathbf{v}-\nabla p & =\mathbf{0}, \\
\nabla \cdot \mathbf{v} & =0 .
\end{aligned}
$$

Here, $C$ is the solute concentration, $\mathbf{v}$ is the fluid velocity, and $p$ is the dynamic pressure.

The governing Eqs. [2.1] and [2.2] in the outer region satisfy the following boundary conditions at the solid "surface" (outer limit of the thin interfacial layer) obtained by solving for the solute concentration and fluid velocity in the inner region and using a matching procedure to ensure a continuous solution in the whole fluid phase $(5,14)$,

$$
\begin{aligned}
\mathbf{n} \cdot \nabla C & =-\beta \mathbf{I}: \nabla_{\mathrm{s}} \nabla_{\mathrm{s}} C, \\
\mathbf{v} & =\mathbf{v}^{*}-\frac{k T}{\eta} L^{*} K \nabla_{\mathrm{s}} C,
\end{aligned}
$$

where the relaxation coefficient

$$
\beta=(1+v \mathrm{Pe}) K
$$




$$
\begin{aligned}
K & =\int_{0}^{\infty}\left[\exp \left(-\Phi\left(y_{n}\right) / k T\right)-1\right] d y_{n}, \\
L^{*} & =K^{-1} \int_{0}^{\infty} y_{n}\left[\exp \left(-\Phi\left(y_{n}\right) / k T\right)-1\right] d y_{n}, \\
v & =\left(L^{*} K^{2}\right)^{-1} \int_{0}^{\infty}\left\{\int_{y_{n}}^{\infty}\left[\exp \left(-\Phi\left(y_{n}^{\prime}\right) / k T\right)-1\right] d y_{n}^{\prime}\right\}^{2} d y_{n},
\end{aligned}
$$

and

$$
\mathrm{Pe}=\frac{k T}{\eta D} L^{*} K C
$$

In Eqs. [2.3]-[2.6], $\Phi$ represents the potential energy resulting from the interaction between a single solute molecule and the fluid/solid interface, $D$ is the solute diffusion coefficient, $y_{n}$ (or $\left.y_{n}^{\prime}\right)$ is the distance measured from the interface along $\mathbf{n}$, which is the unit vector outwardly normal to the interface, $\mathbf{I}$ is the unit dyadic, $\nabla_{\mathrm{s}}=(\mathbf{I}-\mathbf{n n}) \cdot \nabla$ denotes the gradient operator along the interface, and $\mathbf{v}^{*}$ is the velocity of the interface, equal to zero for the fixed solid boundary and $\mathbf{U}+\boldsymbol{\Omega} \times \mathbf{r}$ for a particle translating at velocity $\mathbf{U}$ at a reference point and rotating at angular velocity $\boldsymbol{\Omega}$, where $\mathbf{r}$ is the position vector on the particle surface measured from the reference point. The dimensionless coefficient $v$ is of order 1 and $\nu \mathrm{Pe}$ accounts for the effect of convection on the solute distribution just outside the interfacial layer. The physical meaning of Eq. [2.3] is that the net tangential solute flux along the solid surface must be balanced by the normal solute flux occurring just beyond the diffuse layer to prevent accumulation of the solute. The apparent slip velocity given by the second term on the right-hand side of Eq. [2.4] results from the "diffusio-osmotic" flow caused by the solute-surface interaction energy $\Phi\left(y_{n}\right)$ and the tangential gradient $\nabla_{\mathrm{s}} C$ along the solid surface. To obtain Eqs. [2.3]-[2.6], it was assumed that the concentration of solute within the adsorption boundary layer is related to the solutesurface interaction energy by a Boltzmann distribution (5).

The solute concentration far away from the particle approaches the undisturbed distribution $C_{\infty}$. If there is no osmotic flow generated by the fixed solid boundary, the fluid is motionless far from the particle.

Because there is no effective external field exerted beyond the outer edge of the particle-solute interaction layer, the particle (plus its adjacent diffuse layer of solute molecules) is force and torque free. With this constraint, one can calculate the particle velocities $\mathbf{U}$ and $\boldsymbol{\Omega}$ after solving Eqs. [2.1] and [2.2] for the solute concentration and fluid velocity. For a spherical particle undergoing diffusiophoresis or a circular cylindrical particle undergoing transverse diffusiophoresis in an unbounded solution, the particle velocities can be determined through the above procedure and the result is given by Eq. [1.2] with $\boldsymbol{\Omega}=\mathbf{0}$. Note that the relaxation coefficient $\beta$ in Eq. [1.2] is taken to be a constant determined by Eq. [2.5] with the local solute concentration at the particle surface (outer boundary of the thin diffuse layer) in Eq. [2.6d] equal to the value measured at the particle center in the absence of the particle, which is valid when the fluid is only slightly nonuniform in the undisturbed solute concentration $C_{\infty}$ on the length scale of the particle radius $a$.

\section{DIFFUSIOPHORESIS OF A CIRCULAR CYLINDER NORMAL TO A PLANE WALL}

In this section we consider the diffusiophoretic motion of a long cylindrical particle of radius $a$ in the direction perpendicular to its axis and to a large plane wall located at a distance $d$ from the axis, as illustrated in Fig. 1a. The concentration gradient of the solute far away from the cylinder, $\nabla C_{\infty}$, is a constant equal to $E_{\infty} \mathbf{e}_{x}$, where $\mathbf{e}_{x}$ is a unit vector in the rectangular coordinate system $(x, y, z)$. It is assumed that $L / a^{*} \ll 1$, where $L$ is the characteristic thickness of the layer of interaction between the solute molecules and each solid surface (which is expected to be approximately the molecular dimension of the solute, say $1 \mathrm{~nm}$ ) and $a^{*}$ is the smaller of $a$ and $d-a$. The interaction between the solute molecules and the solid surface is described by the potential energy $\Phi\left(y_{n}\right)$, which decays to zero when $y_{n} / L \gg 1$. It is well known that in transverse motion of a long cylinder end effects modify the solute concentration distribution and flow

(a)

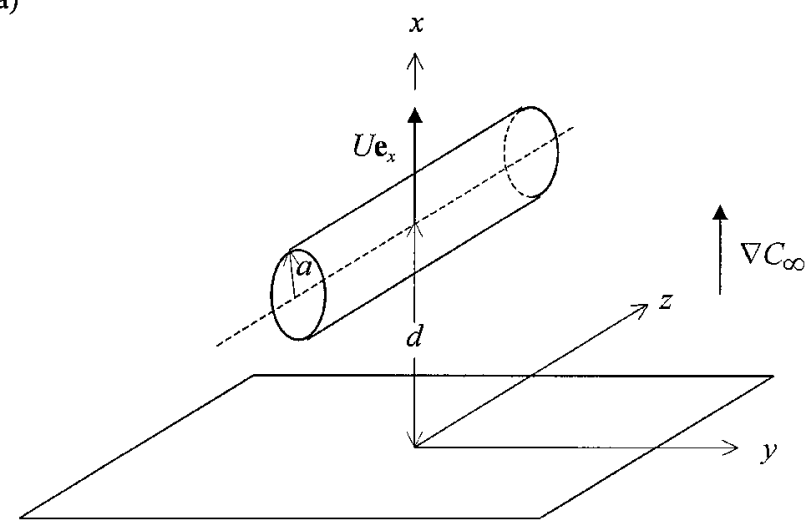

(b)

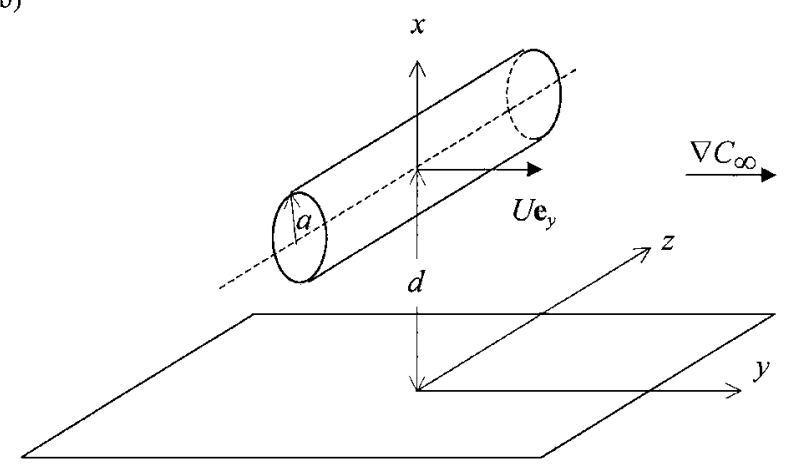

FIG. 1. Geometrical sketch for the transverse diffusiophoresis of a cylinder in the proximity of a plane wall: (a) motion perpendicular to the wall; (b) motion parallel to the wall. 


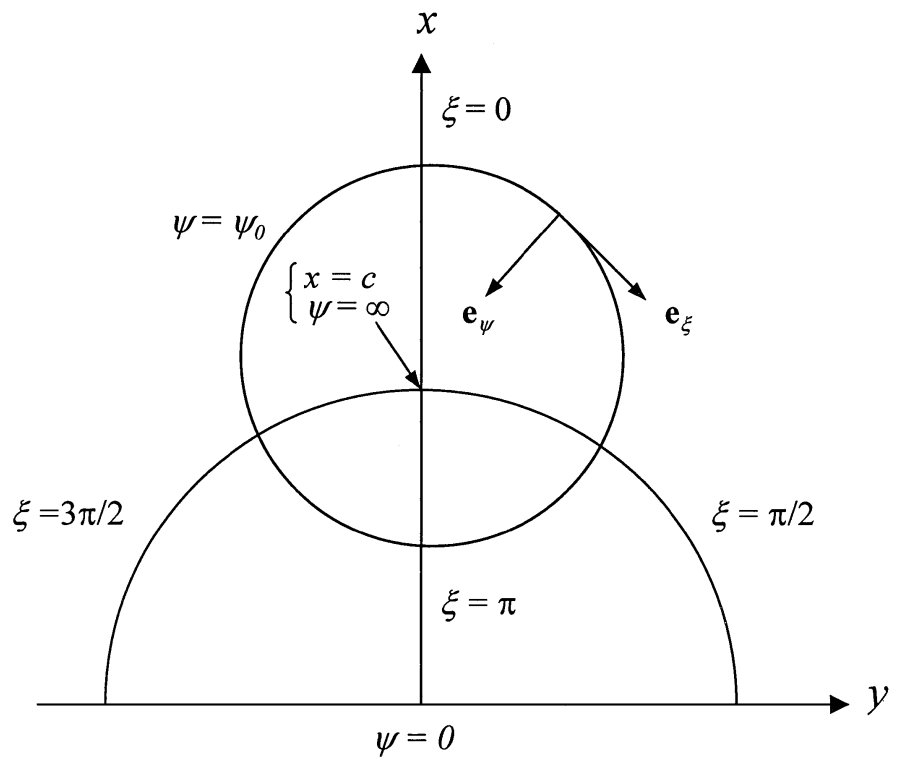

FIG. 2. The two-dimensional bipolar coordinates $(\xi, \psi)$ and rectangular coordinates $(x, y)$.

pattern at large distance from the axis of the cylinder. However, near the cylinder and not too close to the ends, the mass transfer and fluid flow are essentially perpendicular to the cylinder axis. Hence, it may be treated as a two-dimensional problem in a cross section perpendicular to the cylinder. Our purpose is to determine the correction to Eq. [1.2] for the diffusiophoretic velocity of the cylinder due to the presence of the plane wall.

For convenience in satisfying the boundary conditions at solid surfaces, an orthogonal curvilinear coordinate system $(\xi, \psi, z)$ known as cylindrical bipolar coordinates, shown in Fig. 2, is utilized to solve this problem. This coordinate system is related to rectangular coordinates in any plane $z=$ constant by the relation $(15,16)$

$$
\begin{aligned}
& x=\frac{c \sinh \psi}{\cosh \psi-\cos \xi}, \\
& y=\frac{c \sin \xi}{\cosh \psi-\cos \xi},
\end{aligned}
$$

where $-\infty<\psi<\infty, 0 \leq \xi<2 \pi$, and $c$ is a characteristic length in the bipolar coordinate system which is positive.

The curves $\psi=$ constant correspond to a family of nonintersecting, coaxial circles (or cylinders) whose centers all lie along the $x$-axis. The special case $\psi=0$ generates a circle of infinite radius and corresponds to the entire $y$-axis (or the plane $x=0$ ). $\psi=\psi_{0}>0$ represents the circle (or the cylinder) of radius $a=c$ $\operatorname{csch} \psi_{0}$, with its center at the point $\left(x=d=c \operatorname{coth} \psi_{0}, y=0\right)$. The ratio of the radius of the cylinder to the distance of the axis of the cylinder from the plane is related to $\psi_{0}$ by

$$
\lambda=a / d=\operatorname{sech} \psi_{0} .
$$

To determine the diffusiophoretic velocity of the cylinder near the plane wall, it is necessary to ascertain the fluid velocity distribution in the fluid phase. Because the boundary conditions of the fluid velocity field are coupled with the solute concentration gradient at the particle surface, it is necessary to determine the concentration profile first.

\subsection{Solute Concentration Distribution}

The solute concentration $C$ obeys Laplace's equation [2.1] and is subject to the following boundary conditions at the surfaces (or, more precisely, at the outer edges of the thin interfacial layers) of the cylindrical particle (according to Eq. [2.3]) and of the plane wall, and far away from the cylinder:

$$
\begin{aligned}
\frac{\partial C}{\partial \psi} & =\frac{\beta}{c} \frac{\partial}{\partial \xi}\left[(\cosh \psi-\cos \xi) \frac{\partial C}{\partial \xi}\right] \text { at } \psi=\psi_{0}, \\
C & =C_{0}-E_{\infty} d \quad \text { at } \psi=0(\text { or } x=0), \\
C & =C_{0}+E_{\infty}(x-d) \quad \text { as }\left(x^{2}+y^{2}\right)^{1 / 2} \rightarrow \infty \text { and } x \geq 0,
\end{aligned}
$$

where $C_{0}$ is the prescribed solute concentration at the position of the cylinder axis (in the absence of the cylinder). The concentration at the plane wall has been set equal to a constant (so Eq. [2.3] does not apply to allow a uniform solute gradient in the $x$ direction far away from the cylinder). The relaxation coefficient $\beta$ in Eq. [3.3a] is given by Eq. [2.5] with $C$ in Eq. [2.6d] equal to $C_{0}$.

A general solution to Eq. [2.1] suitable for satisfying Eq. [3.3] is (17)

$$
\begin{aligned}
C= & C_{0}+E_{\infty}(x-d) \\
& +E_{\infty} c \sum_{n=1}^{\infty}\left[\left(R_{n} \cosh n \psi+S_{n} \sinh n \psi\right) \cos n \xi\right. \\
& \left.+\left(R_{n}^{\prime} \cosh n \psi+S_{n}^{\prime} \sinh n \psi\right) \sin n \xi\right] .
\end{aligned}
$$

Applying boundary conditions [3.3], one can obtain the following relations for the unknown coefficients in Eq. [3.4].

$$
R_{n}=R_{n}^{\prime}=S_{n}^{\prime}=0,
$$

and

$$
\begin{aligned}
S_{n+1} & {\left[\frac{\beta}{2 c}(n+1) \sinh (n+1) \psi_{0}\right] } \\
& -S_{n}\left[\cosh n \psi_{0}+\frac{\beta}{c} n \cosh \psi_{0} \sinh n \psi_{0}\right] \\
& +S_{n-1}\left[\frac{\beta}{2 c}(n-1) \sinh (n-1) \psi_{0}\right] \\
= & 2 e^{-n \psi_{0}}\left(\frac{\beta}{c} \sinh \psi_{0}-1\right),
\end{aligned}
$$


for $n \geq 1$ with the convention $S_{0}=0$. Because the coefficients $S_{n}$ should approach zero as $n \rightarrow \infty$ for the solute concentration field to remain bounded, they can be determined by simultaneously solving the first $N$ equations of the recurrence relation [3.5b], provided that $N$ is sufficiently large that $S_{N+1}$ is negligible. In the limit $\beta / a=0$ (no polarization of the diffuse layer surrounding the particle), Eq. [3.5b] reduces to a simple explicit formula for $S_{n}$,

$$
S_{n}=4\left(e^{2 n \psi_{0}}+1\right)^{-1} .
$$

\subsection{Fluid Velocity Distribution}

With knowledge of the solution for the solute concentration distribution in the fluid phase, we can now proceed to find the flow field. The velocity distribution for the fluid outside the thin particle-solute interaction layer is governed by the Stokes equations [2.2] or the following fourth-order differential equation for two-dimensional slow viscous flow:

$$
\nabla^{4} \Psi=\nabla^{2}\left(\nabla^{2} \Psi\right)=0
$$

Here, the stream function $\Psi$ is related to the velocity components in bipolar coordinates by

$$
\begin{aligned}
\nu_{\xi} & =\frac{1}{c}(\cosh \psi-\cos \xi) \frac{\partial \Psi}{\partial \psi}, \\
\nu_{\psi} & =-\frac{1}{c}(\cosh \psi-\cos \xi) \frac{\partial \Psi}{\partial \xi},
\end{aligned}
$$

and the operator $\nabla^{2}$ has the from

$$
\begin{aligned}
\nabla^{2} & =\frac{\partial^{2}}{\partial x^{2}}+\frac{\partial^{2}}{\partial y^{2}} \\
& =\frac{1}{c^{2}}(\cosh \psi-\cos \xi)^{2}\left(\frac{\partial^{2}}{\partial \xi^{2}}+\frac{\partial^{2}}{\partial \psi^{2}}\right) .
\end{aligned}
$$

The boundary conditions for the velocity field, resulting from Eq. [2.4] and the fact that the fluid is at rest far away from the cylindrical particle, are

$$
\begin{aligned}
& \mathbf{v}=U \mathbf{e}_{x}-\frac{k T}{\eta} L^{*} K \nabla_{\mathrm{s}} C \quad \text { at } \psi=\psi_{0}, \\
& \mathbf{v}=\mathbf{0} \quad \text { at } \psi=0 \\
& \mathbf{v} \rightarrow \mathbf{0} \quad \text { as }\left(x^{2}+y^{2}\right)^{1 / 2} \rightarrow \infty \text { and } x \geq 0
\end{aligned}
$$

where $U$ is the instantaneous diffusiophoretic velocity of the cylinder to be determined. There will be no rotation of the cylinder due to the symmetry of the flow field about the $x$-axis and no slip velocity along the planar surface since the solute concentration is constant there. In Eq. [3.10a], $\nabla_{\mathrm{s}} C$ can be obtained from the solute concentration distribution given by Eq. [3.4] with coefficients determined from Eq. [3.5].
Because the governing equation and the boundary conditions are linear, the total flow can be decomposed into two parts. First, we consider the fluid velocity field $\mathbf{v}_{1}$ about a cylinder (at $\psi=\psi_{0}$ ) translating perpendicular to its axis and to a plane wall (at $\psi=0$ ) with a velocity $U \mathbf{e}_{x}$ but with no slip velocity at the solid surfaces. The stream function for this creeping flow was obtained $(18,19)$ and the drag force per unit length exerted by the fluid on the cylinder is

$$
\mathbf{F}_{1}=-\frac{4 \pi \eta U}{\psi_{0}-\tanh \psi_{0}} \mathbf{e}_{x}
$$

There is no torque acting on the translating cylinder.

Next, we consider the fluid motion caused by the slip velocity at the surface of a stationary cylinder near a plane wall; namely, the flow subject to the boundary conditions given by Eq. [3.10] with $U$ equal to zero. Superposing this velocity field $\mathbf{v}_{2}$ with $\mathbf{v}_{1}$ will yield the total velocity field produced by the transverse diffusiophoretic motion of a cylinder normal to a plane wall. By obtaining the force exerted on the stationary cylinder, adding it to the force given by Eq. [3.11], and equating the sum to zero, the diffusiophoretic velocity of the cylinder with wall corrections will result.

A general solution to the biharmonic equation [3.7] in bipolar coordinates, suitable for satisfying boundary conditions on the cylinder and plane, has been given by Jeffery (15) and Wakiya (20),

$$
\begin{aligned}
\Psi_{2}= & U_{0} c(\cosh \psi-\cos \xi)^{-1} \\
& \times\{A \psi(\cosh \psi-\cos \xi)+(B+\bar{C} \psi) \sinh \psi-\bar{D} \psi \sin \xi \\
& +\sum_{n=1}^{\infty}\left[a_{n} \cosh (n+1) \psi+b_{n} \sinh (n+1) \psi\right. \\
& \left.+c_{n} \cosh (n-1) \psi+d_{n} \sinh (n-1) \psi\right] \cos n \xi \\
& +\sum_{n=1}^{\infty}\left[a_{n}^{\prime} \cosh (n+1) \psi+b_{n}^{\prime} \sinh (n+1) \psi\right. \\
& \left.\left.+c_{n}^{\prime} \cosh (n-1) \psi+d_{n}^{\prime} \sinh (n-1) \psi\right] \sin n \xi\right\}, \quad \text { 3.12] }
\end{aligned}
$$

where we set $U_{0}=k T L^{*} K E_{\infty} / \eta$, which is a characteristic velocity of the diffusiophoretic particle. The coefficients $A$, $B, \bar{C}, \bar{D}, a_{n}, b_{n}, c_{n}, d_{n}, a_{n}^{\prime}, b_{n}^{\prime}, c_{n}^{\prime}$, and $d_{n}^{\prime}\left(d_{1}\right.$ and $d_{1}^{\prime}$ are trivial $)$ should be determined by the boundary conditions [3.10] with $U=0$ using Eqs. [3.4], [3.5a], and [3.8]. The procedure is straightforward but tedious, with the results

$$
\begin{aligned}
& A=B=\bar{C}=0, \\
& \bar{D}=\frac{\left(2 e^{-\psi_{0}}+S_{1} \cosh \psi_{0}-2 S_{2} \cosh \psi_{0}\right) \sinh ^{3} \psi_{0}}{1+\psi_{0} \sinh 2 \psi_{0}-\cosh 2 \psi_{0}},
\end{aligned}
$$


$a_{n}=b_{n}=c_{n}=d_{n}=0 \quad(n \geq 1)$,

$a_{1}^{\prime}=\frac{2 \psi_{0}-\sinh 2 \psi_{0}}{2\left(\cosh 2 \psi_{0}-1\right)} \bar{D}$,

$b_{1}^{\prime}=\frac{1}{2} \bar{D}, \quad c_{1}^{\prime}=-a_{1}^{\prime}$,

$a_{n}^{\prime}=\frac{(n+1) \sinh (n-1) \psi_{0}-(n-1) \sinh (n+1) \psi_{0}}{8\left(\sinh ^{2} n \psi_{0}-n^{2} \sinh ^{2} \psi_{0}\right)}$

$\times\left[4 e^{-n \psi_{0}} \sinh \psi_{0}+2 n S_{n} \sinh n \psi_{0} \cosh \psi_{0}-(n-1) S_{n-1}\right.$

$\left.\times \sinh (n-1) \psi_{0}-(n+1) S_{n+1} \sinh (n+1) \psi_{0}\right]$

$$
(n \geq 2) \text {, }
$$

$b_{n}^{\prime}=\frac{(n-1)\left[\cosh (n+1) \psi_{0}-\cosh (n-1) \psi_{0}\right]}{(n+1) \sinh (n-1) \psi_{0}-(n-1) \sinh (n+1) \psi_{0}} a_{n}^{\prime}$

$(n \geq 2)$,

$c_{n}^{\prime}=-a_{n}^{\prime}, \quad d_{n}^{\prime}=-\frac{n+1}{n-1} b_{n}^{\prime} \quad(n \geq 2)$.

The force per unit length acting on the stationary cylinder due to the fluid flow caused by the slip velocity at the surface of the cylinder is

$$
\mathbf{F}_{2}=4 \pi \eta U_{0} \bar{D} \mathbf{e}_{x},
$$

where the coefficient $\bar{D}$ is given by Eq. [3.13b].

\subsection{Derivation of the Particle Velocity}

Since the net force exerted on the diffusiophoretic cylinder must vanish, we have

$$
\mathbf{F}_{1}+\mathbf{F}_{2}=\mathbf{0}
$$

To determine the velocity $U$ of the cylinder near the plane wall, the above equation must be solved after substituting Eqs. [3.11] and [3.14] into it. The result is

$$
U=U_{0} \frac{\left(2 e^{-\psi_{0}}+S_{1} \cosh \psi_{0}-2 S_{2} \cosh \psi_{0}\right) \sinh ^{2} \psi_{0}}{2 \cosh \psi_{0}},
$$

which shows that only the coefficients $S_{1}$ and $S_{2}$ contribute to the diffusiophoretic velocity of the cylinder. By the linearity of the problem, the same magnitude of the particle velocity is predicted for a given separation $\lambda$, which is related to $\psi_{0}$ by Eq. [3.2], whether the particle is approaching the plane wall or receding from it.

In the limit $\beta / a=0$ (no polarization of the diffuse layer surrounding the particle), Eq. [3.6] can be substituted into Eq. [3.16] to yield

$$
U=U_{0} \frac{2 e^{-\psi_{0}} \sinh ^{2} \psi_{0}}{\cosh \psi_{0}}\left(1-e^{-\psi_{0}} \frac{\cosh \psi_{0}}{\cosh 2 \psi_{0}}\right)
$$

This closed-form formula can also be used to express the corresponding electrophoretic velocity of a dielectric cylinder with $U_{0}$ representing the value of this velocity (given by the well-known Smoluchowski equation) when the conducting plane wall does not exist. In the results of an earlier analysis for the boundary effects on electrophoresis of a colloidal cylinder with a thin unpolarized double layer (19), the factor $\cosh \psi_{0} / \cosh 2 \psi_{0}$ in the parentheses was inadvertently omitted.

Since the analytical solution for the case of $\beta / a=0$ has been obtained in Eq. [3.17], it is interesting to examine the asymptotic behavior of the particle motion for the situations of wide separation $(\lambda \rightarrow 0)$ and near contact $(\lambda \rightarrow 1)$ between the cylinder and the plane wall. The approximate expressions for the diffusiophoretic velocity resulting from Eq. [3.17] are

$$
\begin{aligned}
& \frac{U}{U_{0}} \rightarrow 1-\lambda^{2} \quad \text { as } \lambda \rightarrow 0, \\
& \frac{U}{U_{0}} \rightarrow 8(1-\lambda)^{2} \quad \text { as } \lambda \rightarrow 1 .
\end{aligned}
$$

It is revealed from Eq. [3.18a] that the wall correction to the diffusiophoretic velocity of the cylinder is $O\left(\lambda^{2}\right)$, which is different from that obtained for a diffusiophoretic sphere, in which the leading wall correction is $O\left(\lambda^{3}\right)$ (in the latter case $\lambda$ is the ratio of the sphere radius to the distance of the sphere center from the wall). For the diffusiophoresis of a sphere of radius $a$ normal to a plane wall, it can be shown that

$$
\frac{U}{U^{(0)}} \rightarrow 1-\frac{5+2 \beta / a}{8(1+\beta / a)} \lambda^{3} \quad \text { as } \lambda \rightarrow 0,
$$

where $U^{(0)}$ is the diffusiophoretic velocity of the sphere in the absence of the wall given by Eq. [1.2].

\subsection{Results and Discussion}

The coefficients $S_{n}$ of the solute concentration distribution [3.4] in the present quasisteady problem have been calculated for various values of the parameters $\beta / a$ and $\lambda$ using a personal computer. For the case of $\beta / a=1000$ and $\lambda=0.999, N$ equal to about 150 was sufficiently large that the $(N+1)$ th term of this series of coefficients is negligible and increases in $N$ do not alter the calculated values of $S_{1}$ and $S_{2}$ appreciably.

Numerical values of the normalized diffusiophoretic mobility $U / U^{(0)}$ of the cylinder evaluated from Eq. [3.16] with different values of $\beta / a$ and $\lambda$ are presented in Table 1 and plotted by the solid curves in Fig. 3, where $U^{(0)}$ is the transverse diffusiophoretic velocity of the cylinder in the absence of the plane wall and is given by Eq. [1.2]. These results can be fitted to the following approximate expression valid when the cylinder is wide apart from the plane:

$$
\frac{U}{U^{(0)}} \rightarrow 1-\frac{1+\beta / 2 a}{1+\beta / a} \lambda^{2} \text { as } \lambda \rightarrow 0 .
$$


TABLE I

The N ormalized Velocity of a C ylinder U ndergoing Transverse Diffusiophoresis Normal to a Plane Wall ${ }^{a}$

\begin{tabular}{llll}
\hline & & $U / U^{(0)}$ & \\
\cline { 2 - 4 }$\lambda$ & $\beta / a=0.1$ & $\beta / a=1$ & $\beta / a=10$ \\
\hline 0 & 1 & 1 & 1 \\
0.1 & 0.99044 & 0.99249 & 0.99453 \\
0.2 & 0.96163 & 0.96980 & 0.97797 \\
0.3 & 0.91314 & 0.93145 & 0.94981 \\
0.4 & 0.84424 & 0.87659 & 0.90913 \\
0.5 & 0.75394 & 0.80385 & 0.85438 \\
0.6 & 0.64100 & 0.71111 & 0.78306 \\
0.7 & 0.50415 & 0.59505 & 0.69085 \\
0.8 & 0.34291 & 0.45000 & 0.56941 \\
0.9 & 0.16113 & 0.26464 & 0.39799 \\
0.95 & 0.06924 & 0.14860 & 0.27268 \\
0.99 & 0.00787 & 0.03488 & 0.10937 \\
0.995 & 0.00292 & 0.01814 & 0.07326 \\
0.999 & 0.00028 & 0.00383 & 0.02871 \\
\hline
\end{tabular}

${ }^{a}$ The relaxation coefficient $\beta$ and the separation parameter $\lambda$ are defined by Eqs. [2.5] and [3.2], respectively.

Although this asymptotic formula slightly overestimates the exact solution of the cylinder velocity, the error is quite small (less than $1.1 \%$ for $\lambda \leq 0.5$ ). Note that, for the limiting case of $\beta / a=0$, Eq. [3.20] reduces to Eq. [3.18a].

For the corresponding diffusiophoretic motion of a sphere perpendicular to a plane wall, a combined analytical-numerical solution was developed by using a boundary collocation method (13). The wall-corrected reduced diffusiophoretic mobility of the sphere as a function of $\lambda$ (in this case $\lambda$ is the ratio of the sphere radius to the distance of the sphere center from the plane wall) is drawn by the dashed curves in Fig. 3 for comparison. As ex-

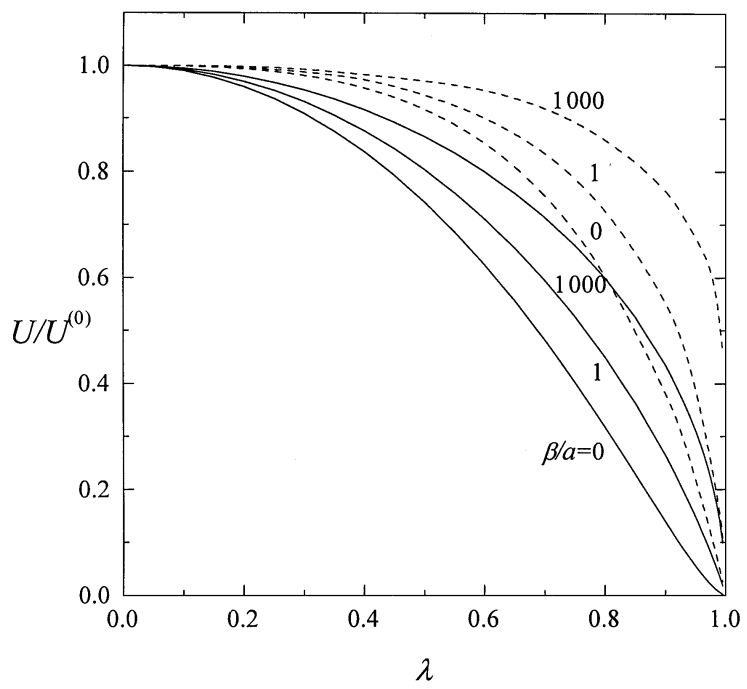

FIG. 3. Plots of the normalized diffusiophoretic mobilities of a cylinder (solid curves, as computed from Eq. [3.16]) and of a sphere (dashed curves, obtained from Keh and Jan (13)) in the direction perpendicular to a plane wall versus $\lambda$ with $\beta / a$ as a parameter. pected, both the cylindrical and the spherical particles will move with the velocity $U^{(0)}$ as $\lambda=0$. The diffusiophoretic velocity of either particle then steadily decreases as the particle approaches the wall (with increasing $\lambda$ ), going to zero at the limit. Also, the retardation effect produced by the wall is a monotonic decreasing function of $\beta / a$. Note that the wall effect on the reduction of the diffusiophoretic mobility for a cylinder (19.6\% for $\beta / a=1$ and $\lambda=0.5)$ is much more significant than that for a sphere (5.4\% for $\beta / a=1$ and $\lambda=0.5$ ). This is because the effective wall-interaction area that offers resistance to the motion of a cylinder is much larger than that to the movement of a sphere.

\section{DIFFUSIOPHORESIS OF A CIRCULAR CYLINDER PARALLEL TO A PLANE WALL}

We now consider the two-dimensional diffusiophoretic motion of a long cylinder of radius $a$ (represented by $\psi=\psi_{0}$ ) in the direction normal to its axis (with coordinates $x=d$ and $y=0$ ) and parallel to a large plane wall (located at $\psi=0$ or $x=0$ ), as shown in Fig. 1b. A constant solute concentration gradient $E_{\infty} \mathbf{e}_{y}$ is imposed. As in the previous section, the assumption of thin interfacial layers is employed. Our objective is to find the wall-corrected diffusiophoretic velocity of the cylindrical particle.

\subsection{Solute Concentration Distribution}

The conservative equation governing the solute concentration $C$ is the Laplace equation [2.1]. Since the concentration gradient far away from the cylinder approaches the applied field and the tangential solute flux is balanced by the normal solute flux at the surface of the particle and the plane wall according to Eq. [2.3], the solute concentration is subject to the boundary conditions

$$
\begin{aligned}
\frac{\partial C}{\partial \psi} & =\frac{\beta}{c} \frac{\partial}{\partial \xi}\left[(\cosh \psi-\cos \xi) \frac{\partial C}{\partial \xi}\right] \quad \text { at } \psi=\psi_{0}, \\
\frac{\partial C}{\partial \psi} & =-\frac{\beta_{w}}{c} \frac{\partial}{\partial \xi}\left[(\cosh \psi-\cos \xi) \frac{\partial C}{\partial \xi}\right] \quad \text { at } \psi=0, \\
C & =C_{0}+E_{\infty} y \quad \text { as }\left(x^{2}+y^{2}\right)^{1 / 2} \rightarrow \infty \text { and } x \geq 0,
\end{aligned}
$$

where $\beta$ and $\beta_{w}$ are the relaxation coefficients at the particle surface and the plane wall, respectively.

The solution form for the solute concentration is still given by Eq. [3.4], but here the term $E_{\infty}(x-d)$ is replaced by $E_{\infty} y$,

$$
\begin{aligned}
C= & C_{0}+E_{\infty} y+E_{\infty} c \sum_{n=1}^{\infty}\left[\left(R_{n} \cosh n \psi+S_{n} \sinh n \psi\right)\right. \\
& \left.\times \cos n \xi+\left(R_{n}^{\prime} \cosh n \psi+S_{n}^{\prime} \sinh n \psi\right) \sin n \xi\right] .
\end{aligned}
$$

Applying Eq. [4.1] to this solution form, one obtains expressions for the coefficients as

$$
R_{n}=S_{n}=0
$$




$$
\begin{aligned}
\frac{\beta}{2 c}(n+1)\left[R_{n+1}^{\prime} \cosh (n+1) \psi_{0}+S_{n+1}^{\prime} \sinh (n+1) \psi_{0}\right] & \\
& -R_{n}^{\prime}\left(\sinh n \psi_{0}+\frac{\beta}{c} n \cosh \psi_{0} \cosh n \psi_{0}\right) \\
& -S_{n}^{\prime}\left(\cosh n \psi_{0}+\frac{\beta}{c} n \cosh \psi_{0} \sinh n \psi_{0}\right) \\
& +\frac{\beta}{2 c}(n-1)\left[R_{n-1}^{\prime} \cosh (n-1) \psi_{0}+S_{n-1}^{\prime} \sinh (n-1) \psi_{0}\right] \\
= & 2 e^{-n \psi_{0}}\left(\frac{\beta}{c} \sinh \psi_{0}-1\right), \\
S_{n}^{\prime}= & \frac{\beta_{w}}{2 c}\left[2 n R_{n}^{\prime}-(n+1) R_{n+1}^{\prime}-(n-1) R_{n-1}^{\prime}\right],
\end{aligned}
$$

for $n \geq 1$ with the convention $R_{0}^{\prime}=S_{0}^{\prime}=0$. Since the coefficients $R_{n}^{\prime}$ and $S_{n}^{\prime}$ become small with a large $n$, simultaneous solution of the first $N$ equations of the recurrence relation [4.3b] after the substitution of Eq. [4.3c] for $S_{n}^{\prime}$ yields $N$ coefficients $R_{n}^{\prime}$, thereby determining the solute concentration according to Eqs. [4.2], [4.3a], and [4.3c]. In the limit $\beta_{w} / a=0$ (no polarization of the diffuse layer adjacent to the plane wall), Eq. [4.3c] becomes

$$
S_{n}^{\prime}=0
$$

If both the relaxation parameters $\beta / a$ and $\beta_{w} / a$ are equal to zero, Eq. [4.3b] reduces to

$$
R_{n}^{\prime}=4\left(e^{2 n \psi_{0}}-1\right)^{-1}
$$

\subsection{Fluid Velocity Distribution}

Having obtained the solution for the solute concentration, we can now proceed to find the fluid velocity field. The fluid motion outside the thin interfacial layers is governed by Eq. [2.2] or [3.7]. At the surfaces of the cylinder and the plane wall, the concentration gradient acting on the diffuse solute within the interfacial layers produces a relative tangential fluid velocity described by Eq. [2.4] at the outer boundary of each interfacial layer. Also, a uniform diffusio-osmotic flow far away from the cylinder is generated by the tangential fluid velocity at the plane wall. Therefore, the boundary conditions for the fluid velocity field are

$$
\begin{aligned}
& \mathbf{v}=U \mathbf{e}_{y}+a \Omega \mathbf{e}_{\xi}-\frac{k T}{\eta} L^{*} K \nabla_{\mathrm{s}} C \quad \text { at } \psi=\psi_{0}, \\
& \mathbf{v}=-\frac{k T}{\eta} L_{\mathrm{w}}^{*} K_{\mathrm{w}} \nabla_{\mathrm{s}} C \quad \text { at } \psi=0, \\
& \mathbf{v} \rightarrow \mathbf{v}_{\infty}=v_{\infty} \mathbf{e}_{y}=-\frac{k T}{\eta} L_{\mathrm{w}}^{*} K_{\mathrm{w}} E_{\infty} \mathbf{e}_{y} \\
& \text { as }\left(x^{2}+y^{2}\right)^{1 / 2} \rightarrow \infty \text { and } x \geq 0,
\end{aligned}
$$

where $K_{\mathrm{w}}$ and $L_{\mathrm{w}}^{*}$ denote the lengths $K$ and $L^{*}$ defined by Eqs. [2.6a,b], respectively, for the interfacial layer at the plane wall, $U$ and $\Omega$ are respectively the translational and angular velocities of the diffusiophoretic cylinder to be determined, and $\nabla_{\mathrm{s}} C$ is obtained from the concentration distribution given by Eqs. [4.2] and [4.3]. Because the cylinder is freely suspended in the fluid, the net force and net torque per unit length exerted by the fluid on the cylinder must vanish.

Analogous to the case dealt with in the previous section, the total flow can be decomposed into two parts. First, we consider the fluid velocity field $\mathbf{v}_{1}$ about a cylinder moving parallel to the plane wall with translational velocity $U \mathbf{e}_{y}$ and angular velocity $\Omega \mathbf{e}_{z}$ but with no slip velocity at the particle surface, while the plane wall and the fluid far from the cylinder are moving with a velocity equal to $\mathbf{v}_{\infty}$. The stream function for this flow was obtained $(18,19)$ and the hydrodynamic force and torque per unit length acting on the cylinder are given by

$$
\begin{aligned}
& \mathbf{F}_{1}=-4 \pi \eta\left(U-v_{\infty}\right) \frac{1}{\psi_{0}} \mathbf{e}_{y}, \\
& \mathbf{T}_{1}=-4 \pi \eta a^{2} \Omega \operatorname{coth} \psi_{0} \mathbf{e}_{z} .
\end{aligned}
$$

It can be seen that the translation and rotation for the twodimensional creeping motion of a circular cylinder parallel to a plane wall are not coupled with each other.

Next, we consider the fluid flow caused by the tangential slip velocities at the surfaces (i.e., outer edges of the diffuse layers) of a stationary cylinder and a nearby plane wall,

$$
\begin{aligned}
& \mathbf{v}_{2}=-\frac{k T}{\eta} L^{*} K \nabla_{\mathrm{s}} C \quad \text { at } \psi=\psi_{0}, \\
& \mathbf{v}_{2}=-\frac{k T}{\eta} L_{\mathrm{w}}^{*} K_{\mathrm{w}} \nabla_{\mathrm{s}} C-\mathbf{v}_{\infty} \quad \text { at } \psi=0, \\
& \mathbf{v}_{2} \rightarrow \mathbf{0} \text { as }\left(x^{2}+y^{2}\right)^{1 / 2} \rightarrow \infty \text { and } x \geq 0 .
\end{aligned}
$$

Superposing the velocity field $\mathbf{v}_{2}$ with $\mathbf{v}_{1}$ yields the total fluid velocity field produced by the transverse diffusiophoretic motion of a cylinder parallel to a plane wall. By obtaining the force and torque exerted by the fluid on the stationary cylinder, individually adding these to the force and torque given by Eq. [4.6], and equating the results to zero, the translational and angular velocities of the cylinder will result.

The stream function $\Psi_{2}$ associated with $\mathbf{v}_{2}$ can also be expressed by Eq. [3.12], and the coefficients $A, B, \bar{C}, \ldots$, etc. should be determined by applying Eq. [4.7] to Eq. [3.12] and using Eqs. [3.8], [4.2], and [4.3a]. After considerable algebraic manipulation, one obtains

$$
\begin{aligned}
\bar{C}= & -\frac{1}{4 \psi_{0}}\left(2 \alpha R_{2}^{\prime}-4 e^{-\psi_{0}} \sinh \psi_{0}-2 R_{1}^{\prime} \cosh ^{2} \psi_{0}\right. \\
& \left.-S_{1}^{\prime} \sinh 2 \psi_{0}+2 R_{2}^{\prime} \cosh 2 \psi_{0}+2 S_{2}^{\prime} \sinh 2 \psi_{0}\right),
\end{aligned}
$$




$$
\begin{aligned}
A= & -\frac{1}{4 \sinh ^{2} \psi_{0}}\left(2 \alpha R_{1}^{\prime}-4 e^{-\psi_{0}} \cosh \psi_{0}\right. \\
& \left.-2 R_{1}^{\prime} \cosh ^{2} \psi_{0}-S_{1}^{\prime} \sinh 2 \psi_{0}\right) \\
& -\frac{\psi_{0}+\sinh \psi_{0} \cosh \psi_{0}}{\sinh ^{2} \psi_{0}} \bar{C}
\end{aligned}
$$

$$
B=-A+\frac{1}{2} \alpha R_{1}^{\prime}, \quad \bar{D}=0,
$$

$a_{1}=-\frac{1}{2} A \tanh \psi_{0}-\frac{1}{4 \sinh 2 \psi_{0}}\left[2 \alpha\left(R_{2}^{\prime}-R_{1}^{\prime}\right) \cosh 2 \psi_{0}\right.$

$$
+4 e^{-\psi_{0}} \sinh \psi_{0}+2 R_{1}^{\prime} \cosh ^{2} \psi_{0}+S_{1}^{\prime} \sinh 2 \psi_{0}
$$$$
\left.-2 R_{2}^{\prime} \cosh 2 \psi_{0}-2 S_{2}^{\prime} \sinh 2 \psi_{0}\right] \text {, }
$$

$$
b_{1}=\frac{1}{2} A+\frac{1}{2} \alpha\left(R_{2}^{\prime}-R_{1}^{\prime}\right), \quad c_{1}=-a_{1},
$$

$$
\begin{aligned}
a_{n}= & \frac{1}{8\left(\sinh ^{2} n \psi_{0}-n^{2} \sinh ^{2} \psi_{0}\right)}\left\{\alpha \left(\sinh 2 n \psi_{0}\right.\right. \\
& \left.-n \sinh 2 \psi_{0}\right)\left[2 n R_{n}^{\prime}-(n+1) R_{n+1}^{\prime}-(n-1) R_{n-1}^{\prime}\right] \\
& +\left[(n-1) \sinh (n+1) \psi_{0}-(n+1) \sinh (n-1) \psi_{0}\right] \\
& \times\left[4 e^{-n \psi_{0}} \sinh \psi_{0}+2 n \cosh \psi_{0}\left(R_{n}^{\prime} \cosh n \psi_{0}\right.\right. \\
& \left.+S_{n}^{\prime} \sinh n \psi_{0}\right)-(n+1)\left(R_{n+1}^{\prime} \cosh (n+1) \psi_{0}\right. \\
& \left.+S_{n+1}^{\prime} \sinh (n+1) \psi_{0}\right)-(n-1)\left(R_{n-1}^{\prime} \cosh (n-1) \psi_{0}\right. \\
& \left.\left.\left.+S_{n-1}^{\prime} \sinh (n-1) \psi_{0}\right)\right]\right\} \quad(n \geq 2),
\end{aligned}
$$

$$
\begin{aligned}
b_{n}= & \frac{(n-1)\left[\cosh (n-1) \psi_{0}-\cosh (n+1) \psi_{0}\right]}{(n-1) \sinh (n+1) \psi_{0}-(n+1) \sinh (n-1) \psi_{0}} a_{n} \\
& +\frac{\alpha \sinh (n-1) \psi_{0}}{2\left[(n-1) \sinh (n+1) \psi_{0}-(n+1) \sinh (n-1) \psi_{0}\right]} \\
& \times\left[2 n R_{n}^{\prime}-(n+1) R_{n+1}^{\prime}-(n-1) R_{n-1}^{\prime}\right] \quad(n \geq 2),
\end{aligned}
$$

$$
\begin{aligned}
c_{n}= & -a_{n} \quad(n \geq 2), \\
d_{n}= & -\frac{n+1}{n-1} b_{n}-\frac{\alpha}{2(n-1)} \\
& \times\left[2 n R_{n}^{\prime}-(n+1) R_{n+1}^{\prime}-(n-1) R_{n-1}^{\prime}\right] \quad(n \geq 2),
\end{aligned}
$$

$$
a_{n}^{\prime}=b_{n}^{\prime}=c_{n}^{\prime}=d_{n}^{\prime}=0 \quad(n \geq 1),
$$

where the parameter $\alpha=L_{\mathrm{w}}^{*} K_{\mathrm{w}} / L^{*} K=-v_{\infty} / U_{0}$.

The force and torque per unit length exerted on the stationary cylinder by the fluid due to the flow caused by the slip velocities at the surfaces of the cylinder and the plane wall are

$$
\begin{aligned}
& \mathbf{F}_{2}=4 \pi \eta U_{0} \bar{C} \mathbf{e}_{y}, \\
& \mathbf{T}_{2}=-4 \pi \eta a U_{0}\left(A \sinh \psi_{0}+\bar{C} \cosh \psi_{0}\right) \mathbf{e}_{z},
\end{aligned}
$$

where the coefficients $\bar{C}$ and $A$ are given by Eqs. [4.8a,b].

\subsection{Derivation of the Particle Velocities}

Since the net hydrodynamic force and torque acting on the diffusiophoretic cylinder vanish, we have

$$
\begin{gathered}
\mathbf{F}_{1}+\mathbf{F}_{2}=\mathbf{0}, \\
\mathbf{T}_{1}+\mathbf{T}_{2}=\mathbf{0} .
\end{gathered}
$$

To determine the translational velocity $U$ and angular velocity $\Omega$ of the cylinder near a plane wall, the above equations must be solved after substituting Eqs. [4.6] and [4.9] into them. The results are

$$
\begin{aligned}
U & =U_{0} \bar{C} \psi_{0}+v_{\infty}, \\
a \Omega & =-U_{0} \sinh \psi_{0}\left(A \tanh \psi_{0}+\bar{C}\right) .
\end{aligned}
$$

From Eqs. [4.8a,b] for the coefficients $A$ and $\bar{C}$ and Eq. [4.3c], one can see that only the coefficients $R_{1}^{\prime}, R_{2}^{\prime}$, and $R_{3}^{\prime}$ make a contribution to the velocities of the diffusiophoretic cylinder. The connection between $\psi_{0}$ and the separation parameter $\lambda$ is given by Eq. [3.2].

In the limit $\beta / a=\beta_{\mathrm{w}} / a=0$ (no polarization of the interfacial layers), Eq. [4.4] can be substituted into Eqs. [4.8a,b] to compute $\bar{C}$ and $A$, and Eq. [4.11] leads to

$$
\begin{aligned}
U & =U_{0}(1-\alpha) \operatorname{coth} 2 \psi_{0}, \\
a \Omega & =-U_{0}(1-\alpha)\left(\frac{\operatorname{sech} \psi_{0}}{\sinh 2 \psi_{0}}\right) .
\end{aligned}
$$

These closed-form formulas are identical to the result obtained by Keh et al. (19) for the transverse electrophoresis of a nonconducting cylinder parallel to a dielectric plane. Note that, in this limit, the direction of the particle motion is simply determined by the value of $1-\alpha$.

The asymptotic expressions for the particle velocities under the situations of wide separation $(\lambda \rightarrow 0)$ and near contact $(\lambda \rightarrow 1)$ between the cylinder and the plane derived from Eq. [4.12] are

$$
\begin{aligned}
& \frac{U}{U_{0}(1-\alpha)} \rightarrow 1+\frac{1}{8} \lambda^{4} \quad \text { as } \lambda \rightarrow 0, \\
& \frac{U}{U_{0}(1-\alpha)} \rightarrow 2^{-3 / 2}(1-\lambda)^{-1 / 2} \quad \text { as } \lambda \rightarrow 1 ; \\
& \frac{a \Omega}{U_{0}(1-\alpha)} \rightarrow-\frac{1}{2} \lambda^{3} \quad \text { as } \lambda \rightarrow 0,
\end{aligned}
$$




$$
\frac{a \Omega}{U_{0}(1-\alpha)} \rightarrow-2^{-3 / 2}(1-\lambda)^{-1 / 2} \quad \text { as } \lambda \rightarrow 1
$$

In general, for the transverse diffusiophoresis of a long cylinder, the boundary effect on the particle translation is $O\left(\lambda^{2}\right)$, as shown by Eqs. [3.18a] and [3.20] for the motion perpendicular to the plane wall. However, the coefficient of the $\lambda^{2}$ term in Eq. [4.13a] turns out to be zero, and the leading term of wall correction for the cylinder translation in the limit $\beta / a=\beta_{\mathrm{w}} / a=0$ becomes $O\left(\lambda^{4}\right)$ when the motion is parallel to the plane wall. Note that, for a diffusiophoretic cylinder that allows free rotation about its axis, the leading term of the particle angular velocity caused by the neighboring boundary is of $O\left(\lambda^{3}\right)$, as illustrated in Eq. [4.14a]. On the other hand, for a sphere moving parallel to a plane wall by diffusiophoresis (with free rotation) for the case of $\beta / a=\beta_{\mathrm{w}} / a=0$, it was shown by a method of reflections that $(11,21)$

$$
\begin{aligned}
& \frac{U}{U_{0}(1-\alpha)}=1-\frac{1}{16} \lambda^{3}+\frac{1}{8} \lambda^{5}-\frac{25}{256} \lambda^{6}+O\left(\lambda^{8}\right), \\
& \frac{a \Omega}{U_{0}(1-\alpha)}=-\frac{3}{16} \lambda^{4}+\frac{99}{256} \lambda^{7}+O\left(\lambda^{9}\right) .
\end{aligned}
$$

It can be seen that the wall corrections to the translational and angular velocities of a diffusiophoretic sphere are of the orders $O\left(\lambda^{3}\right)$ and $O\left(\lambda^{4}\right)$, respectively, in contrast to those $\left(O\left(\lambda^{2}\right)\right.$ and $O\left(\lambda^{3}\right)$, respectively) for a long cylinder undergoing transverse diffusiophoresis.

When $\lambda=0$ (the parallel plane wall is very far from the diffusiophoretic cylinder), Eq. [4.11] becomes

$$
\begin{aligned}
U & =U^{(0)}+v_{\infty}=U^{(0)}\left[1-\alpha\left(1+\frac{\beta}{a}\right)\right], \\
a \Omega & =0 .
\end{aligned}
$$

Equation [4.16a] represents a superposition of the undisturbed diffusiophoretic velocity of the particle given by Eq. [1.2] and the uniform diffusio-osmotic flow due to the interaction between the applied solute gradient and the plane wall expressed by Eq. [4.5c].

\subsection{Results and Discussion}

The coefficients $R_{n}^{\prime}$ of the solute concentration distribution [4.2] have been calculated for various values of the parameters $\beta / a, \beta_{\mathrm{w}} / a, \alpha$, and $\lambda$ using a personal computer. For the most time-consuming cases, $N$ equal to about 1000 was employed such that the $(N+1)$ th term of this series of coefficients is negligible and an increase in $N$ does not change the calculated values of $R_{1}^{\prime}, R_{2}^{\prime}$, and $R_{3}^{\prime}$ appreciably.

Under the particular situation of $\beta_{\mathrm{w}} / a=0$ and $\alpha=0$ (no polarization of the interfacial layer and no diffusio-osmotic flow at the plane wall), the numerical results of the normalized translational velocity $U / U^{(0)}$ and rotational velocity $a \Omega / U^{(0)}$ of the (a)

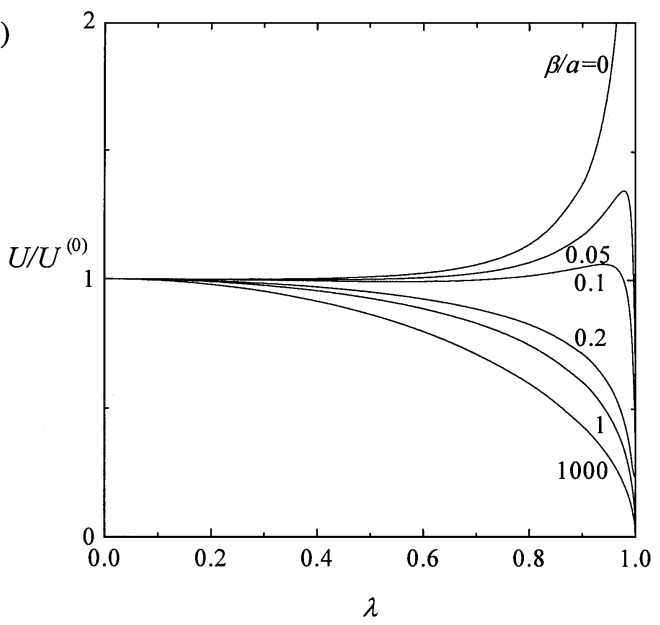

(b)

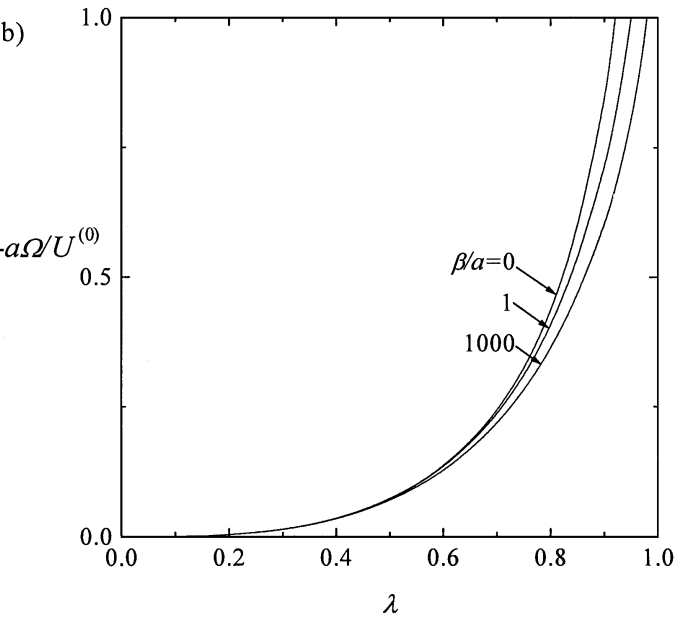

FIG . 4. Plots of the normalized velocities of a cylinder undergoing transverse diffusiophoresis parallel to a plane wall with $\beta_{\mathrm{w}} / a=0$ and $\alpha=0$ versus $\lambda$ with $\beta / a$ as a parameter: (a) translational velocity; (b) rotational velocity.

diffusiophoretic cylinder evaluated from Eq. [4.11] with different values of $\beta / a$ and $\lambda$ are presented in Table 2 and depicted in Fig. 4. Certainly, the cylinder moves (without rotation) with the velocity $U^{(0)}$ given by Eq. [1.2] as $\lambda=0$. When $\beta / a=0$, the diffusiophoretic mobility of the cylinder is a monotonic increasing function of $\lambda$ and becomes infinity at the limit of $\lambda \rightarrow 1$. This behavior is understandable because the local solute concentration gradient at the particle surface on the near side to the plane wall is enhanced (due to the crowding of the solute diffusion lines when they squeeze between the particle and the wall) in comparison with that on the far side. The influence of this enhancement on the particle velocity in the case of $\beta / a=0$ is very important and much stronger than the effect of viscous retardation caused by the wall. When $\beta / a$ is finite but small (say, less than 0.1), the diffusiophoretic mobility of the cylinder is not a monotonic function of $\lambda$, and the value of $U / U^{(0)}$ can be either greater or less than unity depending on the competition between the solute-gradient enhancement and the viscous retardation produced by the wall to the diffusiophoretic motion of the 
TABLE II

The N ormalized Translational and R otational Velocities of a Cylinder U ndergoing Transverse D iffusiophoresis Parallel to a Plane Wall with $\beta_{\mathrm{w}} / a=0$ and $\alpha=0$

\begin{tabular}{|c|c|c|c|c|c|c|}
\hline \multirow[b]{2}{*}{$\lambda$} & \multicolumn{3}{|c|}{$U / U^{(0)}$} & \multicolumn{3}{|c|}{$-a \Omega / U^{(0)}$} \\
\hline & $\beta / a=0.1$ & $\beta / a=1$ & $\beta / a=10$ & $\beta / a=0.1$ & $\beta / a=1$ & $\beta / a=10$ \\
\hline 0 & 1 & 1 & 1 & 0 & 0 & 0 \\
\hline 0.1 & 0.99955 & 0.99749 & 0.99544 & 0.00050 & 0.00050 & 0.00050 \\
\hline 0.2 & 0.99827 & 0.98979 & 0.98159 & 0.00409 & 0.00408 & 0.00407 \\
\hline 0.3 & 0.99638 & 0.97643 & 0.95791 & 0.01417 & 0.01415 & 0.01402 \\
\hline 0.4 & 0.99431 & 0.95644 & 0.92340 & 0.03501 & 0.03485 & 0.03428 \\
\hline 0.5 & 0.99287 & 0.92820 & 0.87641 & 0.07247 & 0.07180 & 0.06988 \\
\hline 0.6 & 0.99353 & 0.88888 & 0.81424 & 0.13575 & 0.13334 & 0.12792 \\
\hline 0.7 & 0.99913 & 0.83323 & 0.73221 & 0.24170 & 0.23348 & 0.21957 \\
\hline 0.8 & 1.01535 & 0.74999 & 0.62118 & 0.42878 & 0.40002 & 0.36541 \\
\hline 0.9 & 1.05089 & 0.60712 & 0.45777 & 0.82769 & 0.70720 & 0.61554 \\
\hline 0.95 & 1.06153 & 0.47586 & 0.33168 & 1.30404 & 0.99587 & 0.83085 \\
\hline 0.99 & 0.87034 & 0.24715 & 0.15246 & 2.52781 & 1.49066 & 1.16174 \\
\hline 0.995 & 0.72461 & 0.18145 & 0.10840 & 3.00633 & 1.62894 & 1.24664 \\
\hline 0.999 & 0.40537 & 0.08518 & 0.04866 & 3.83403 & 1.82782 & 1.36361 \\
\hline
\end{tabular}

particle. When $\beta / a$ is relatively large (say, greater than 0.2 ), the diffusiophoretic mobility of the cylinder becomes a monotonic decreasing function of $\lambda$. Obviously, in this case, the effect of the viscous drag of the wall is stronger than the wall effect on the interaction between the particle and the solute gradient. As long as the value of $\beta / a$ is finite, the diffusiophoretic mobility of the cylinder tends to vanish at the limit of $\lambda \rightarrow 1$. On the other hand, the normalized rotational velocity of the diffusiophoretic cylinder is a monotonic increasing function of $\lambda$ for any given value of $\beta / a$. The direction of rotation is opposite to that for a sphere sedimenting in the same direction as the diffusiophoresis parallel to the plane [it has been shown that a circular cylinder migrating parallel to a plane wall under a body-force field does not rotate $(18,19)]$. Note that, for a fixed value of $\lambda$, both the values of $U / U^{(0)}$ and $-a \Omega / U^{(0)}$ decrease with the increase of $\beta / a$.

For the special situation of $\beta_{\mathrm{w}}=\beta$ and $\alpha=1$ (the same surface properties of the particle and the plane wall), the numerical results of $U / U^{(0)}$ and $a \Omega / U^{(0)}$ of the diffusiophoretic cylinder with various values of $\beta / a$ and $U / U^{(0)}$ are presented in Table 3 and depicted in Fig. 5. Due to the existence of the uniform diffusio-osmotic flow far from the cylinder, the ratio $U / U^{(0)}$ is no longer equal to unity as $\lambda=0$, but is given by Eq. [4.16a]. When $\beta / a=0$, both the translational and the rotational velocities of

TABLE III

The N ormalized Translational and R otational Velocities of a Cylinder U ndergoing Transverse D iffusiophoresis Parallel to a Plane Wall with $\beta_{\mathrm{w}}=\beta$ and $\alpha=1$

\begin{tabular}{|c|c|c|c|c|c|c|}
\hline \multirow[b]{2}{*}{$\lambda$} & \multicolumn{3}{|c|}{$U / U^{(0)}$} & \multicolumn{3}{|c|}{$-a \Omega / U^{(0)}$} \\
\hline & $\beta / a=0.1$ & $\beta / a=1$ & $\beta / a=10$ & $\beta / a=0.1$ & $\beta / a=1$ & $\beta / a=10$ \\
\hline 0 & 0.1 & 1 & 10 & 0 & 0 & 0 \\
\hline 0.1 & 0.10052 & 1.00251 & 9.99774 & 0.00006 & 0.00050 & 0.00251 \\
\hline 0.2 & 0.10243 & 1.01021 & 9.98864 & 0.00053 & 0.00408 & 0.01487 \\
\hline 0.3 & 0.10641 & 1.02357 & 9.97380 & 0.00207 & 0.01414 & 0.04200 \\
\hline 0.4 & 0.11362 & 1.04356 & 9.95306 & 0.00580 & 0.03485 & 0.08901 \\
\hline 0.5 & 0.12612 & 1.07180 & 9.92484 & 0.01377 & 0.07180 & 0.16281 \\
\hline 0.6 & 0.14805 & 1.11111 & 9.88568 & 0.03010 & 0.13333 & 0.27408 \\
\hline 0.7 & 0.18867 & 1.16676 & 9.82840 & 0.06421 & 0.23347 & 0.44160 \\
\hline 0.8 & 0.27363 & 1.25000 & 9.73588 & 0.14261 & 0.40000 & 0.70505 \\
\hline 0.9 & 0.50499 & 1.39286 & 9.55053 & 0.37260 & 0.70716 & 1.18141 \\
\hline 0.95 & 0.84075 & 1.52410 & 9.33918 & 0.71957 & 0.99579 & 1.64860 \\
\hline 0.99 & 1.79233 & 1.75275 & 8.80101 & 1.71890 & 1.49044 & 2.60192 \\
\hline 0.995 & 2.16491 & 1.81839 & 8.56789 & 2.11019 & 1.62859 & 2.94773 \\
\hline 0.999 & 2.79626 & 1.91441 & 8.06091 & 2.77061 & 1.82699 & 3.61531 \\
\hline
\end{tabular}


(a)

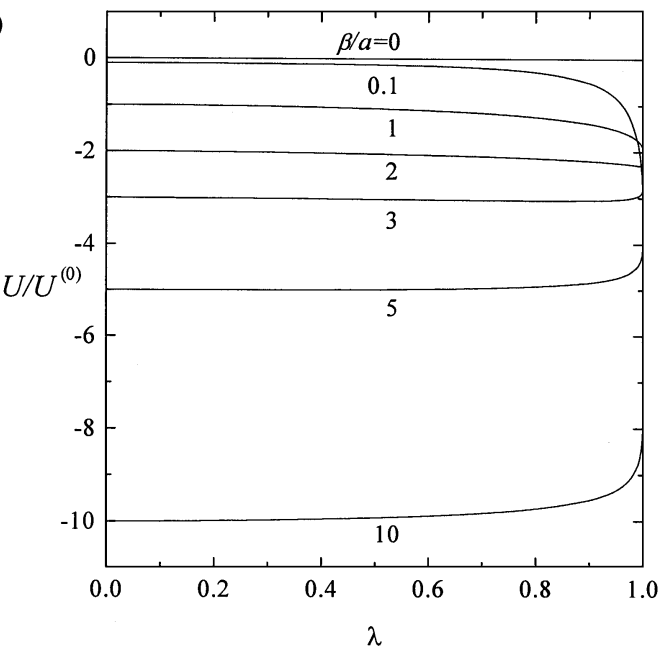

(b)

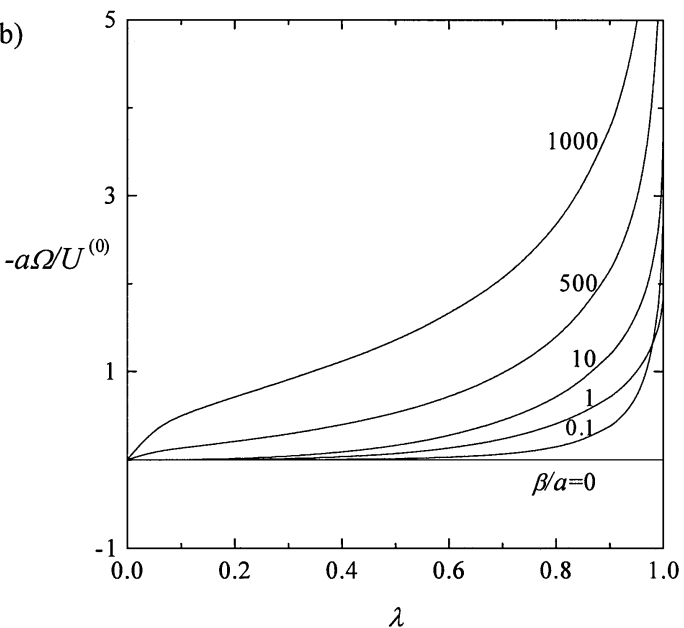

FIG. 5. Plots of the normalized velocities of a cylinder undergoing transverse diffusiophoresis parallel to a plane wall with $\beta_{\mathrm{w}}=\beta$ and $\alpha=1$ versus $\lambda$ with $\beta / a$ as a parameter: (a) translational velocity; (b) rotational velocity.

the particle vanish, irrespective of the value of $\lambda$. For any finite value of $\beta / a$, the particle translates in the direction opposite to that which would occur if it is undergoing diffusiophoresis in an unbounded fluid $\left(U / U^{(0)}<0\right)$, but the particle still rotates along the same direction as in the situation of $\beta_{\mathrm{w}} / a=0$ and $\alpha=0$. The value of $U / U^{(0)}$ is a monotonic decreasing function of $\lambda$ if $\beta / a$ is small (say, less than 2) and is a monotonic increasing function of $\lambda$ if $\beta / a$ is large (say, greater than 5). On the other hand, the value of $-a \Omega / U^{(0)}$ is always a monotonic increasing function of $\lambda$ for any finite value of $\beta / a$. It can be seen that the value of $U / U^{(0)}$ decreases and the value of $-a \Omega / U^{(0)}$ increases with the increase of $\beta / a$ for a given value of $\lambda$ which is not too close to unity.

In Figs. 6 and 7, plots of the numerical results of $U / U^{(0)}$ and $a \Omega / U^{(0)}$ of the diffusiophoretic cylinder versus $\lambda$ under the situation of $\beta_{\mathrm{w}}=\alpha \beta$ with $\alpha=0.5$ and 2, respectively, are drawn for various values of $\beta / a$. Again, Eq. [4.16] predicts the limiting case of $\lambda=0$. Figure 6 depicts a typical situation of $0<\alpha<1$ (with the two bounds shown in Figs. 4 and 5). When $\beta / a=0, U / U^{(0)}$ is always a positive value and increases monotonically with the increase of $\lambda$. For a given finite value of $\beta / a$, this normalized translational velocity may not be a monotonic function of $\lambda$. When $\beta / a>\alpha^{-1}-1$, the value of $U / U^{(0)}$ is always negative. For an arbitrary value of $\beta / a,-a \Omega / U^{(0)}$ is always a positive value and increases monotonically with the increase of $\lambda$.

Figure 7 represents a typical situation of $\alpha>1$, in which the value of $U / U^{(0)}$ always negative. When $\beta / a=0,-a \Omega / U^{(0)}$ is always a negative value and decreases monotonically with the increase of $\lambda$. When $\beta / a$ is finite but small, this normalized rotational velocity is not a monotonic function of $\lambda$ and its value can be either positive or negative. When $\beta / a$ is relatively large, $-a \Omega / U^{(0)}$ is always a positive value and increases monotonically with the increase of $\lambda$.

(a)

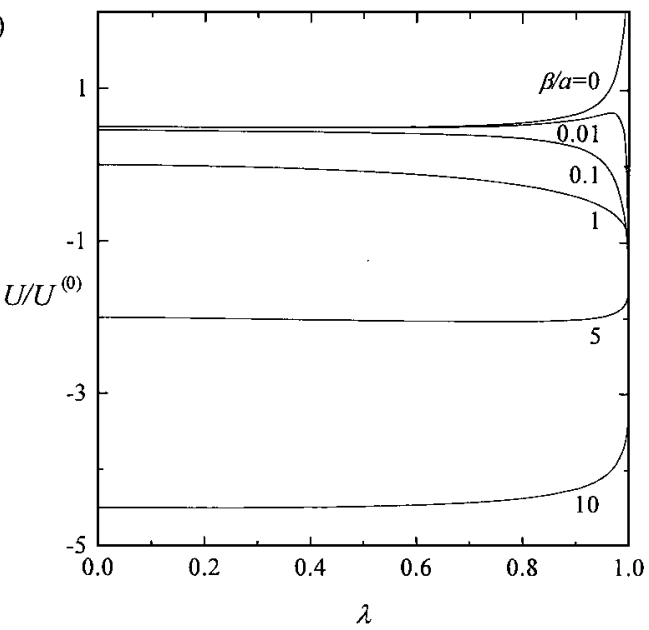

(b)

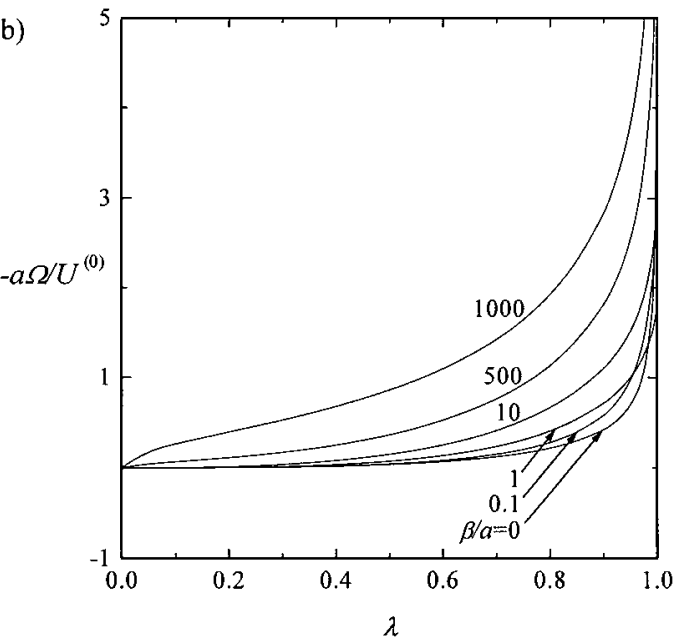

FIG. 6. Plots of the normalized velocities of a cylinder undergoing transverse diffusiophoresis parallel to a plane wall with $\beta_{\mathrm{w}}=\alpha \beta$ and $\alpha=0.5$ versus $\lambda$ with $\beta / a$ as a parameter: (a) translational velocity; (b) rotational velocity. 
(a)

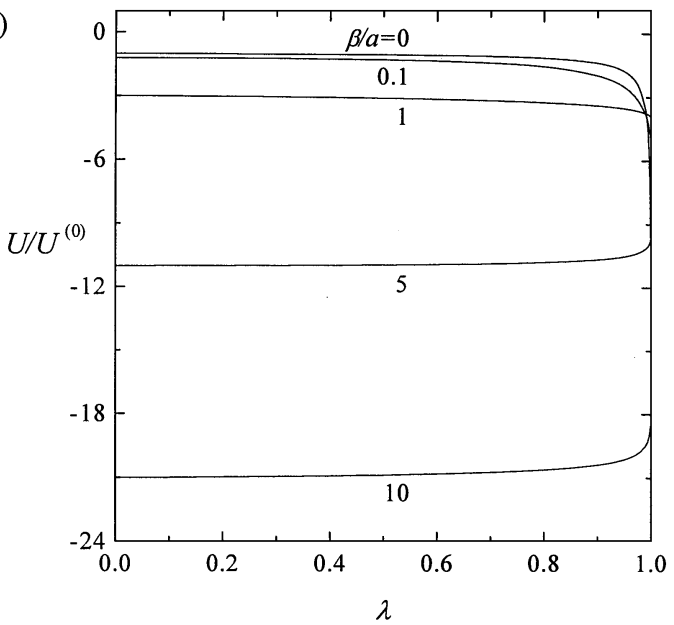

(b)

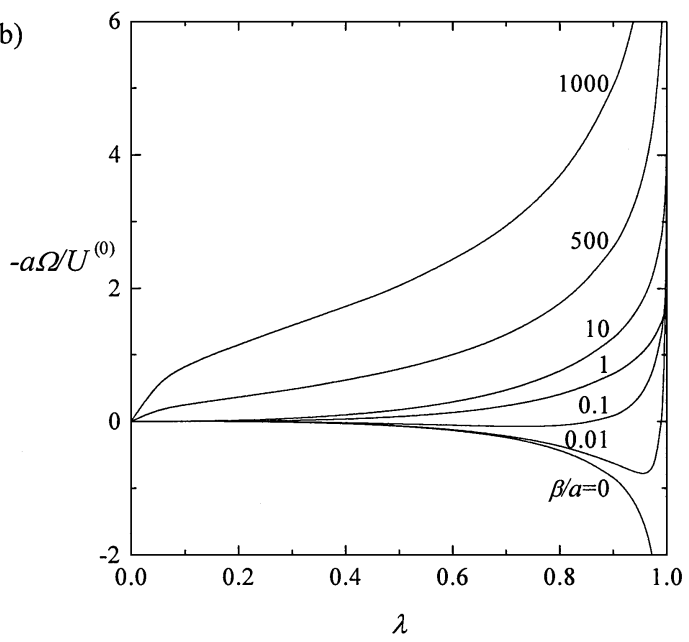

FIG. 7. Plots of the normalized velocities of a cylinder undergoing transverse diffusiophoresis parallel to a plane wall with $\beta_{\mathrm{w}}=\alpha \beta$ and $\alpha=2$ versus $\lambda$ with $\beta / a$ as a parameter: (a) translational velocity; (b) rotational velocity.

\section{SU MMARY}

In this work the solutions for the problem of two-dimensional diffusiophoresis of a long circular cylinder in a nonelectrolyte gradient in the proximity of a large plane wall have been obtained using cylindrical bipolar coordinates in two fundamental cases: motion normal to the plane and motion parallel to the plane. Although the region of the interaction between the solute and the solid surfaces is taken to be small compared to the particle radius and to the spacing between the particle and the wall, the assumption allows for the polarization of the diffuse solute in the interfacial layers. Because the governing equations and the boundary conditions for the solute concentration distribution and fluid flow field in the "outer" region applicable to the general problem of two-dimensional diffusiophoresis of a circular cylinder in an arbitrary direction with respect to a plane wall are linear, the solution for the general problem can be obtained as a superposition of the solutions for its two fundamental subproblems considered in Sections 3 and 4.
When the solute gradient is applied perpendicular to a plane wall, the diffusiophoretic mobility of the particle decreases monotonically as the particle approaches the wall (with the increase of the separation parameter $\lambda$ ) and goes to zero at the limit. The larger the polarization effect in the interfacial layer surrounding the particle (or the value of the relaxation parameter $\beta / a$ ), the weaker the wall effect on the diffusiophoresis. For any given values of $\beta / a$ and $\lambda$, the boundary effect on the diffusiophoretic motion of a cylinder is much stronger than that of a sphere. On the other hand, when the solute gradient is imposed parallel to the plane wall, the interaction between the diffusiophoretic cylinder and the wall is a complicated function of the relevant parameters due to the effect of polarization of the diffuse layer adjacent to the plane and the existence of the uniform diffusio-osmotic flow far from the particle produced by the tangential fluid velocity at the wall. In general, the influence of the parallel wall on the diffusiophoretic motion of the particle with the consideration of the polarization effect in the diffuse layers is quite different (both quantitatively and qualitatively) from that without taking account of this effect. For some situations, the normalized translational and rotational velocities of the diffusiophoretic particle do not vary monotonically with $\lambda$. So far, there are no results available in the literature for the diffusiophoresis of a spherical particle parallel to a plane wall involving the polarization effect in the diffuse layers to compare with our results for a circular cylinder.

\section{ACKN OWLEDG MENTS}

Part of this research was supported by the National Science Council of the Republic of China. The authors thank Mr. J. S. Jan and Mr. H. J. Tu for the help with some mathematical work.

\section{REFERENCES}

1. Dukhin, S. S., and Derjaguin, B. V., in "Surface and Colloid Science" (E. Matijevic, Ed.), Vol. 7. Wiley, New York, 1974.

2. Ebel, J. P., Anderson, J. L., and Prieve, D. C., Langmuir 4, 396 (1988).

3. Staffeld, P. O., and Quinn, J. A., J. Colloid Interface Sci. 130, 88 (1989).

4. Anderson, J. L., Lowell, M. E., and Prieve, D. C., J. Fluid Mech. 117, 107 (1982).

5. Anderson, J. L., and Prieve, D. C., Langmuir 7, 403 (1991).

6. Keh, H. J., and Huang, T. Y., J. Colloid Interface Sci. 160, 354 (1993).

7. Keh, H. J., and Chen, S. B., Langmuir 9, 1142 (1993).

8. Keh, H. J., and Huang, T. Y., Colloid Polym. Sci. 272, 855 (1994).

9. Anderson, J. L., Annu. Rev. Fluid Mech. 21, 61 (1989).

10. Morrison, F. A., and Stukel, J. J., J. Colloid Interface Sci. 33, 88 (1970).

11. Keh, H. J., and Anderson, J. L., J. Fluid Mech. 153, 417 (1985).

12. Keh, H. J., and Chiou, J. Y., AIChE J. 42, 1397 (1996).

13. Keh, H. J., and Jan, J. S., J. Colloid Interface Sci. 183, 458 (1996).

14. O’Brien, R. W., J. Colloid Interface Sci. 92, 204 (1983).

15. Jeffery, G. B., Proc. R. Soc. London A 101, 169 (1922).

16. Happel, J., and Brenner, H., "Low Reynolds Number Hydrodynamics." Nijhoff, The Netherlands, 1983.

17. Umemura, A., J. Fluid Mech. 121, 345 (1982).

18. Jeffrey, D. J., and Onishi, Y., Q. J. Mech. Appl. Maths 34, 129 (1981).

19. Keh, H. J., Horng, K. D., and Kuo, J., J. Fluid Mech. 231, 211 (1991).

20. Wakiya, S., J. Phys. Soc. Jpn. 39, 1603 (1975).

21. Keh, H. J., unpublished notes (1998). 